\title{
The application of close incisional negative pressure wound therapy in revision arthroplasty among asian patients: a comparative study
}

\author{
Ping Keung Chan ${ }^{1 *} \mathbb{D}$, Wing Chiu Fung ${ }^{2}$, Kar Hei Lam² ${ }^{2}$ Winnie Chan³ ${ }^{3}$ Vincent Wai Kwan Chan ${ }^{1}$, Henry Fu', \\ Amy Cheung ', Man Hong Cheung ${ }^{2}$, Chun Hoi Yan² and Kwong Yuen Chiu ${ }^{2}$
}

\begin{abstract}
Introduction: Peri-prosthetic joint infection (PJI) was one of the main causes of revision of arthroplasty. In order to reduce wound complications and surgical site infections, close incisional negative pressure wound therapy (ciNPWT) has been introduced into arthroplasty. This study was designed to review the clinical benefits of the application of ciNPWT in revision arthroplasty.

Methods: This was a single-centre retrospective comparative study approved by the Institutional Review Board. Patients, who underwent revision total knee arthroplasty or revision total hip arthroplasty at the author's institution from January 2016 to October 2019, were included in this study. The ciNPWT cohort included all eligible patients, who underwent operations from January 2018 to October 2019, with the use of ciNPWT $(n=36)$. The control cohort included all eligible patients, who underwent operations from January 2016 to December 2017 with the use of conventional dressing $(n=48)$. The incidences of wound complications were compared to both cohorts.

Results: There was a statistically significant difference in the rate of superficial surgical site infection (SSI) between control cohort and ciNPWT cohort (12.5\% in control vs $0 \%$ in ciNPWT, $p=0.035)$. However, there was no statistically significance of the overall wound complication rate for both cohorts. (14.6\% in control vs $8.3 \%$ in ciNPWT, $p=0.504)$.

Conclusions: The application of ciNPWT could result in a lower rate of superficial surgical site infection when compared with conventional dressing among the patients undergoing revision total knee and total hip arthroplasties.

Trial registration: UW19-706

Keywords: Closed-incisional negative pressure wound therapy, Surgical site infection, Periprosthetic joint infection, Arthroplasty
\end{abstract}

\section{Introduction}

Complications to wound healing can be detrimental to the clinical outcomes after arthroplasty. It can lead to major complications including infection and need for subsequent surgery. Among patients having primary

*Correspondence: cpk464@yahoo.com.hk

${ }^{1}$ Department of Orthopaedics and Traumatology, Queen Mary Hospital, Hong Kong SAR, China

Full list of author information is available at the end of the article total knee arthroplasties, $0.33 \%$ had early wound complications, which subsequently resulted in further surgical management within 30 days [1]. Those patients requiring surgery for wound complications from the first 30 days had 7-13 times higher risk of deep infection when compared to those without wound problems [1, 2]. Measures to prevent wound complications were even more important to revision arthroplasty as revision total knee arthroplasty was associated with a 15 fold increase 
in postoperative complications rates relative to primary knee arthroplasty [3].

Various measures were advocated to optimize wound healing after arthroplasty. These included preoperative optimization of modifiable risk factors such as nutritional status, smoking status, and diabetic control. Intraoperatively, surgeon should have meticulous handling of the skin and soft tissue. Advanced dressing management is another important area, which has recently got more attention as more scientific evidence proven it clinical effectiveness in arthroplasty.

Traditionally, cotton gauze fabric was widely used in wound care because of its excellent absorption power and low cost. However, other advanced dressing materials were recently showed to have more meaningful clinical benefits. The application of a commercial silverimpregnated occlusive dressing, which is a hydrofiber dressing with antibi-microbial ionic silver, was showed to result in a fourfold reduction in acute periprosthetic joint infection when compared with conventional sterile gauze dressing [4]. Closed-incisional negative pressure wound therapy (ciNPWT) is another evidence-based option, which was put in the place of field of arthroplasty for over 10 years [5]. A recent randomized controlled trial comparing the effectiveness of ciNPWT versus silver-impregnated dressing showed that ciNPWT was statistically significant more effective in reducing the 90-day postoperative surgical site complication (ciNPWT: $3.4 \%$ vs silver-dressing: $14.3 \%$; odds ratio (OR): $0.22 ; p=0.0013$ ) [6].

The proposed primary mechanisms of ciNPWT in promoting wound healing include: (i) macrodeformation by drawing wound edges together, (ii) microdeformation by facilitating cell proliferation, and (iii) fluid removal from extra-cellar matrix and environmental control to keep the wound insulated, warm and hydrated [7]. It has been propose that ciNPWT could better protect incisions against external contamination compared to conventional dressing [8]. Studies were also proposed that ciNPWT facilitates healing by improving perfusion, reducing oedema and preventing the formation of haematoma $[9,10]$. Long-term benefits include improving the incision quality in terms of mechanical strength and histological properties [11-14].

The cost of the application of these advanced dressing materials is still a concern. The cost of single-use, disposable reported in the literature was between $\$ 500$ and $\$ 600$ USD [15]. In Asia, limited literatures were expected to be released to discuss the application of ciNPWT in arthroplasty. One of the reasons of low utilization for ciNPWT in Asia might be due to the cost consideration.

Revision arthroplasty was deemed to be a major risk factor of wound related complications. According to the national registries from England and Finland, revision arthroplasty showed an increased risk of postoperative infection-related complications when compared to primary arthroplasty $[16,17]$. A recent review studying the clinical effectiveness of ciNPWT in arthroplasty showed that ciNPWT was indicated to be used in revision arthroplasty instead of primary arthroplasty because of the relatively higher risk of wound complication in revision arthroplasty [18]. Moreover, the use of ciNPWT for infection prevention following revision total knee arthroplasty was demostrated to be cost-effective [19]. Based upon the cost-effectiveness consideration, ciNPWT was mainly utilized in patients having the high-risk of wound complications (revision THA and revision TKA) since January 2018 in our institution.

This study aimed to review the efficacy of the application of ciNPWT in revision arthroplasty when compared with conventional dressing in the authors' institution, which is one of the major joint replacement centres in Asia.

\section{Methods Study design}

This was a single-centre, retrospective comparative study approved by the Institutional Review Board (HKU/HA HKW IRB) (Reference no. UW19-706) in a tertiary referral university hospital.

Patients, who underwent revision TKA or THA at our institution from January 2016 to October 2019, were included in this study. The conventional dressing used traditionally was Cosmopor ${ }^{\circledR} \mathrm{E}$ (Hartmann), which is a sterile, adhesive wound dressing made of soft, non-woven polyester. Since January 2018, the surgical team changed the dressing to ciNPWT, which were either Prevena Incision Management System (Acelity, San Antonio, TX) or PICO (Single use ciNPWT system, Smith \& Nephew, Hull, UK) (Fig. 1). The change of practice in wound dressing provided us with 2 naturally formed cohort groups: those patients who underwent surgery between January 2016 to December 2017 received a conventional dressing, whereas those patients who underwent surgery between Jan 2018 to October 2019 received ciNPWT. All patients were treated by the same surgical team consisting of four fellowship-trained surgeons, who performed revision surgery with similar surgical techniques.

The patients in both cohorts were managed according to the same perioperative protocols with the difference mainly in the dressing material used at the end of the surgical procedures. Prophylactic antibiotics, single dose of $1 \mathrm{~g}$ cefazolin, were given 30-min before the surgical incision. For revision surgery due to aseptic causes e.g. aseptic loosening or periprosthetic fracture, cefazolin would only be used for $24-\mathrm{h}$ after the operation. For revision surgery because of infection, 2-stage revision 


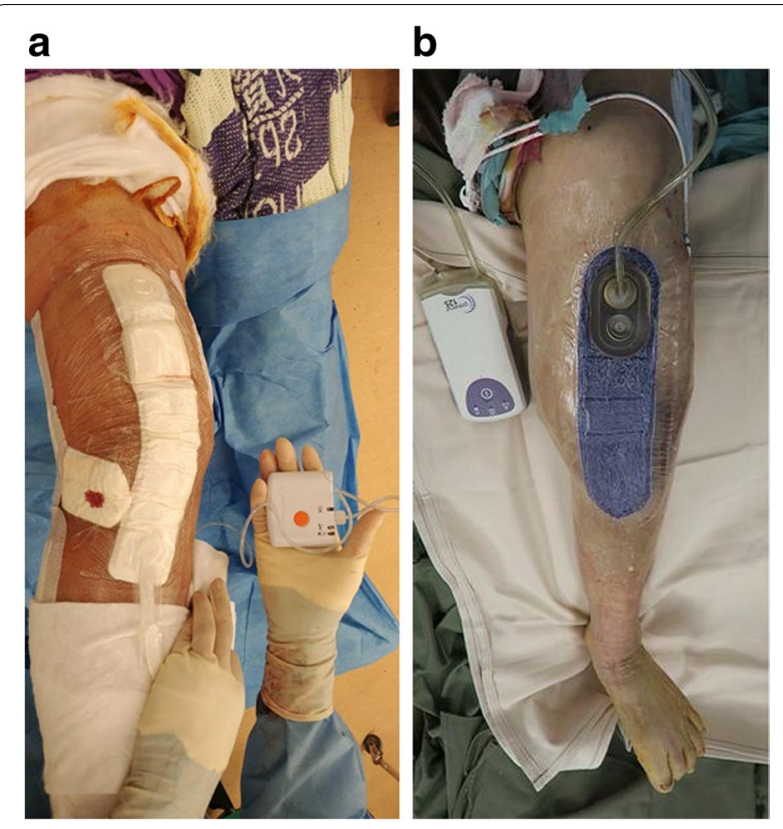

Fig. 1 ciNPWT devices used in revision arthroplasties. a PICO system b Prevena system

surgery would be performed. The perioperative antibiotic used was according to the recommendation by clinical microbiologist based on the antibiotic sensitivity profile of the bacteria. To decrease perioperative blood loss, Intravenous tranexamic acid was given at a dosage of $15 \mathrm{mg} / \mathrm{kg}$ before induction of anaesthesia, and another intravenous injection of the same dosage would be given 4-h afterwards. For revision TKA, tourniquet was used throughout the surgical invasive procedures. Antibioticloaded cement (PALACOS ${ }^{\circledR}+G$, Heraeus), was used for all cemented prosthesis. Allogenic blood transfusion was prescribed if postoperative haemoglobin drop to $\leq 7 \mathrm{~g} / \mathrm{dL}$ in hemodynamically stable adults, or $\leq 8 \mathrm{~g} / \mathrm{dL}$ in patients who had underly cardiovascular or respiratory comorbidities. No surgical drainage was used. All wounds were closed in layers with barbed sutures (Stratafix, Depuy Synthes).

For the postoperative wound surveillance, the dressing in the ciNPWT cohort would be kept intact for 7 days, and then changed to conventional dressing, whereas the wound condition in the conventional dressing cohort was reviewed at postoperative day 3 , and then a new piece of conventional dressing would be re-applied to the surgical wound. The conventional dressing used in both cohorts would be kept intact and removed on postoperative day 14.

All the patient's medical records and perioperative parameters were reviewed. Patient demographics, the type of revision arthroplasty, indications for surgery, mode of anaesthesia, American Society of Anaesthesiologist (ASA) grade and operative duration were recorded. Patients' preoperative status were also documented, including haemoglobin and albumin level, comorbidities and risk factors for wound complications. Clinical photos were taken at the surgical sites with a high-resolution digital camera, and the wound conditions were documented in the wound surveillance chart by a nursing specialist, who specialized in managing surgical wounds in the orthopaedic department. The nurse was in charge of the documentation in the wound surveillance chart, documentation of the postoperative surgical wound conditions, including both septic and aseptic wound complications, during the course of recovery in the in-patient and out-patient periods. Septic complications, such as surgical site infections (SSI) and prosthetic joint infection (PJI) were recorded. SSI, including both superficial incisional SSI and deep incisional SSI, were defined according to the criteria by Centers for Disease Control (CDC) [20], while prosthetic joint infection (PJI) was defined according to the criteria provided by Musculoskeletal Infection Society (MSIS) [21]. Aseptic wound complications, such as persistent drainage, haematoma formation, wound dehiscence, suture granuloma, blister formation, and maceration of the wound, were also documented. The patient would be arranged to have wound surveillance in the out-patient follow-up at 2-week, 6-week, 3-month, 6-month and yearly after discharge from hospital. Data, including wound complications, re-operations due to wound complications of other causes, readmission within 30 days of surgery, periprosthetic joint infection, 90-day perioperative and mortality, were retrieved from the medical record system at our institution.

The primary outcomes were (1) the overall incidence of septic and aseptic wound complications, and (2) the incidence of septic and (3) the incidence of aseptic wound complications. The secondary outcomes include the length of hospital stay, re-operations due to wound complications, 90-day postoperative mortality, and readmission within 30 days of surgery. All the outcome measures were reviewed by two orthopaedic specialists individually, and the cases would be reviewed together so as to make a consensus in case a discrepancy in the individual assessments.

\section{Statistical analysis}

The statistical analysis was performed using IBM SPSS 26.0 software (IBM, Armonk, NY, USA), and the statistically significance took place at the $5 \%$ significance level. The primary and secondary outcome measures was compared. To control for confounding factors, the baseline data between the two cohorts, including patient demographics, existing comorbidities, types of revision 
arthroplasty performed, indications for revision arthroplasty, ASA grade, mode of anaesthesia, operation duration, as well as preoperative and postoperative levels of albumin and haemoglobin, were also compared.

The choice of statistical tests based on the data distribution, and the nature of the data (nominal, ordinal or interval/ratio). The independent samples t test was used for parametric data, while the chi-square or Fisher exact test was used only non-parametric data and categorical data depending on the observed frequency. All available data were incorporated in data listings and tabulations. No imputation of values for missing data were performed.

\section{Results}

A total of 84 patients were reflected in the present study. They underwent either revision hip arthroplasty $(n=38)$ or revision knee arthroplasty $(n=46)$. The majority of cases were revised because of infection $(n=43$, $51.2 \%$ ), whereas the others were revised because of different aseptic causes $(n=41,48.8 \%)$, including loosening, polythene wear, instability, fracture, and dislocation.
36 patients were enrolled in the ciNPWT cohort and 48 patients in the control cohort. Among the ciNPWT cohort, the PICO and Prevena systems were utilized or 22 and 14 patients respectively.

There were no significant differences in terms of age, sex, types of revision procedure performed, indications for revision arthroplasty, ASA grade, mode of anaesthesia, operation duration, as well as preoperative and postoperative levels of albumin and haemoglobin, between the two cohorts (Table 1). While revision arthroplasty itself being a major risk factor for wound complications, some patients had additional risk factors, most commonly history of prior joint infection (ciNPWT $63.9 \%$ Vs Control 41.7\%), ASA grade $\geq 3$ (ciNPWT $61.2 \%$ Vs Control 60.4\%), and diabetes mellitus (ciNPWT 33.3\%Vs Control 20.8\%) (Table 2). Apart from the risk factor of prior joint infection reaching statistically significant (ciNPWT 63.9\% Vs Control 41.7\%, $p=0.05$ ), other risk factors in both cohorts did not show a statistically significant difference.

The bacteriology in PJI cases in both cohorts were reviewed in details (Table 3). There were no statistically

Table 1 Patients demographics and surgical details

\begin{tabular}{|c|c|c|c|c|c|c|}
\hline & & \multicolumn{2}{|l|}{ ciNPWT } & \multicolumn{2}{|l|}{ Control } & \multirow[t]{2}{*}{$P$-value } \\
\hline & & Number of patients & $\%$ & Number of patients & $\%$ & \\
\hline Total Number of patients & & 36 & & 48 & & \\
\hline Age at operations & Mean age & 69.4 & & 70.9 & & 0.557 \\
\hline \multirow[t]{2}{*}{ Sex } & Male & 20 & 55.6 & 17 & 35.4 & 0.066 \\
\hline & Female & 16 & 44.4 & 31 & 64.6 & \\
\hline \multirow[t]{2}{*}{ Types of revision surgery } & Hip & 12 & 33.3 & 26 & 54.2 & 0.058 \\
\hline & Knee & 24 & 66.7 & 22 & 45.8 & \\
\hline \multirow[t]{8}{*}{ Indications } & Infected & 23 & 63.9 & 20 & 41.7 & 0.083 \\
\hline & Loosening & 5 & 13.9 & 17 & 35.4 & \\
\hline & PE wear/ failure & 1 & 2.8 & 4 & 8.3 & \\
\hline & Instability & 1 & 2.8 & 2 & 4.2 & \\
\hline & Wound drainage & 0 & 0 & 2 & 4.2 & \\
\hline & Fracture & 1 & 2.8 & 2 & 4.2 & \\
\hline & Dislocation & 4 & 11 & 1 & 2.1 & \\
\hline & Flexion contracture & 1 & 2.8 & 0 & 0 & \\
\hline \multirow[t]{2}{*}{ ASA Grade } & $\leq 2$ & 15 & 41.7 & 19 & 39.6 & 0.847 \\
\hline & $\geq 3$ & 21 & 58.3 & 29 & 60.4 & \\
\hline \multirow[t]{2}{*}{ Anesthesia } & General / General \& regional & 27 & 75 & 39 & 81.3 & 0.490 \\
\hline & Spinal / Combined spinal epidural & 9 & 25 & 9 & 18.8 & \\
\hline Operation Duration (min) & Mean & 207.2 & & 225.5 & & 0.376 \\
\hline \multirow[t]{3}{*}{ Haemoglobin } & Per-operative mean & 11.5 & & 11.9 & & 0.322 \\
\hline & Post-operative mean & 9.4 & & 9.8 & & 0.306 \\
\hline & Transfusion & 11 & 30.6 & 11 & 22.9 & 0.431 \\
\hline \multirow[t]{2}{*}{ Albumin } & Pre-operative mean & 38.31 & & 39.83 & & 0.243 \\
\hline & Post-operative mean & 28.56 & & 27.77 & & 0.530 \\
\hline
\end{tabular}


Table 2 Patient risk factors / Comorbidities

\begin{tabular}{|c|c|c|c|c|c|}
\hline & \multicolumn{2}{|l|}{ ciNPWT } & \multicolumn{2}{|l|}{ Control } & \multirow[t]{2}{*}{$P$-value } \\
\hline & Number of patients & $\%$ & Number of patients & $\%$ & \\
\hline Total Number & 36 & & 48 & & \\
\hline \multicolumn{6}{|l|}{ Risk Factors } \\
\hline Prior Joint Infection & 23 & 63.9 & 20 & 41.7 & 0.050 \\
\hline$A S A \geq 3$ & 21 & 58.3 & 29 & 60.4 & 0.847 \\
\hline Diabetes Mellitus & 12 & 33.3 & 10 & 20.8 & 0.197 \\
\hline Smoker & 12 & 33.3 & 11 & 22.9 & 0.289 \\
\hline Cardiovascular Disease: IHD / Heart Failure / CABG / PCI before & 10 & 27.8 & 12 & 25.0 & 0.774 \\
\hline Obesity (BMI $\geq 30)$ & 9 & 25.0 & 10 & 20.8 & 0.651 \\
\hline Active Cancer / Previous Cancer with RT to surgical site & 4 & 11.1 & 8 & 16.7 & 0.471 \\
\hline Pre-operative Albumin $\leq 30$ & 4 & 11.1 & 2 & 4.2 & 0.395 \\
\hline Liver Disease & 2 & 5.7 & 1 & 2.1 & 0.570 \\
\hline Renal Failure & 2 & 5.6 & 2 & 4.2 & 1.000 \\
\hline Active Infection / Sepsis & 2 & 5.6 & 3 & 6.3 & 1.000 \\
\hline Depression / Schizophrenia & 2 & 5.6 & 1 & 2.1 & 0.574 \\
\hline Deep Vein Thrombosis & 1 & 2.8 & 1 & 2.1 & 1.000 \\
\hline B12 Deficiency Anaemia & 1 & 2.8 & 1 & 2.1 & 0.676 \\
\hline Current use of corticosteroid / Immunosuppressant & 0 & 0 & 2 & 4.2 & 0.504 \\
\hline
\end{tabular}

BMI Body Mass index, RT Radiotherapy, IHD Ischaemic Heart Disease, CABG Coronary artery bypass graft, PCI Percutaneous coronary intervention, ASA American Society of anesthesiologists

Table 3 Comparison of bacteriology of PJl cases in the cohorts

\begin{tabular}{|c|c|c|c|}
\hline & ciNPWT(\%) & Control(\%) & $P$-value \\
\hline Culture negative PJI & $44 \%$ & $30 \%$ & 0.362 \\
\hline Culture positive PJI & $56 \%$ & $70 \%$ & 0.362 \\
\hline \multicolumn{4}{|l|}{ Among Culture positive PJ } \\
\hline - Methicillin sensitive Staphylococcus aureus & $69.2 \%$ & $78.6 \%$ & 0.454 \\
\hline - Streptococcus & $15.4 \%$ & $0 \%$ & 0.222 \\
\hline - Diphtheroids & $7.7 \%$ & $7.1 \%$ & 0.741 \\
\hline - Other organism & $7.7 \%$ & $14.3 \%$ & 0.529 \\
\hline - Antibiotic resistant organism eg MRSA, ESBL E-coli, VRSA & $0 \%$ & $0 \%$ & 1.000 \\
\hline - With gram positive Bacteria ${ }^{\text {a }}$ & $92.3 \%$ & $100 \%$ & 0.481 \\
\hline - With gram negative Bacteria ${ }^{\text {a }}$ & $15.4 \%$ & $7.1 \%$ & 0.471 \\
\hline -MSSA & $69.2 \%$ & $78.6 \%$ & 0.454 \\
\hline • Non-MSSA & $30.8 \%$ & $21.4 \%$ & 0.454 \\
\hline - PJI with positive culture of 1 bacteria & $92.3 \%$ & $92.9 \%$ & 0.741 \\
\hline - PJI with positive culture $>=2$ bacteria & $7.7 \%$ & $7.1 \%$ & 0.741 \\
\hline
\end{tabular}

MRSA Methicillin-resistant Staphylococcus aureus, ESBL E-coli Extended Spectrum Beta-Lactamase Escherichia coli, VRSA Vancomycin-resistant Staphylococcus aureus, MSSA Methicillin sensitive Staphylococcus aureus

a Not add up to $100 \%$ because the culture results could have 2 bacteria, one gram positive and one gram negative

significant differences in the septic and aseptic revision case distribution in both cohorts (septic revision: ciNPWT 63.9\% Vs Control 41.7\%, $p=0.082$ ). For the septic revision cases, the diagnosis of PJI was based on the MSIS criteria [21]. There was also no statistically significant difference in culture negative PJI in both cohorts
(ciNPWT 44\% Vs Control30\%, $p=0.362$ ). Among PJI cases having a positive bacterial culture, there was no statistically significant difference in the bacteriological profile. Methicillin sensitive staphylococcus aureus (MSSA) was the most commonly found organism in both cohorts, and there were no statistically significant 
differences in case distribution in both cohorts (MSSA: ciNPWT $69.2 \%$ Vs Control: 78.6\%, $p=0.454)$. And there were no antibiotic resistant organisms, eg methicillin resistant staphylococcus aureus, in both cohorts.

Across both cohorts, 10 patients (11.9\%) developed wound complications, including superficial SSI $(n=6)$, persistent drainage $(n=3)$, blister formation $(n=1)$ and haematoma formation requiring drainage $(n=1)$. There was one case of reoperation due to superficial SSI, other cases of superficial SSI were treated with antibiotic only.
The overall wound complication rate of the ciNPWT cohort was $8.3 \%(n=3,2$ persistent drainage, 1 blister formation) compared to $14.6 \%$ for the control cohort ( $n=7,6$ superficial SSIs, 1 persistent drainage). Hence, the overall wound complication rates of the two cohorts did not differ statistical significantly $(p=0.504)$ (Table 4 , Fig. 2). Septic wound complications (SSI and PJI) and aseptic wound complications (persistent drainage, haematoma formation, wound dehiscence, suture granuloma, blister formation, and maceration of the wound) were compared separately. The rate of septic wound

Table 4 Summary of primary and secondary outcomes

\begin{tabular}{|c|c|c|c|c|c|}
\hline & \multicolumn{2}{|l|}{ ciNPWT } & \multicolumn{2}{|l|}{ Control } & \multirow[t]{2}{*}{$P$-value } \\
\hline & Number of patients & $\%$ & Number of patients & $\%$ & \\
\hline Total Number & 36 & & 48 & & \\
\hline \multicolumn{6}{|l|}{ Primary outcomes } \\
\hline Overall wound complications & 3 & 8.3 & 7 & 14.6 & 0.504 \\
\hline Septic wound complications & 0 & 0 & 6 & 12.5 & $0.035^{*}$ \\
\hline Aseptic wound complications & 3 & 8.3 & 1 & 2.5 & 0.309 \\
\hline \multicolumn{6}{|l|}{ Secondary outcomes } \\
\hline Length of hospital stay (Mean) & 31.5 & & 22.9 & & 0.125 \\
\hline Re-operations due to wound complications & 0 & 0 & 1 & 2.1 & 1.000 \\
\hline 90-day perioperative mortality & 0 & 0 & 1 & 2.1 & 1.000 \\
\hline Readmission within 30 days of surgery & 0 & 0 & 0 & 0 & 1.000 \\
\hline
\end{tabular}

${ }^{*}=$ statistical significance at $p<0.05$

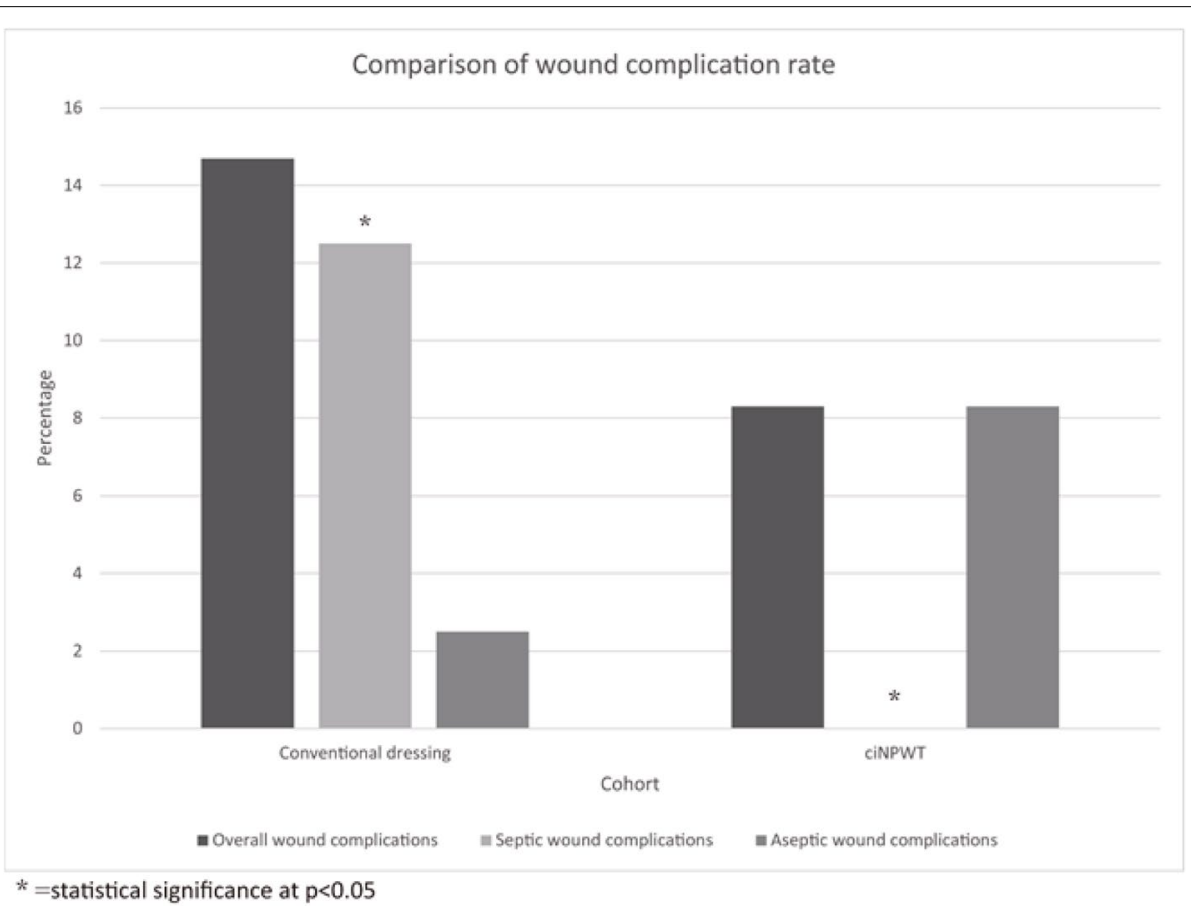

Fig. 2 Comparison of wound complication rate. ${ }^{*}=$ statistical significance at $p<0.05$ 
complication was statistically significantly higher in the control cohort than the ciNPWT cohort (ciNPWT 0\% Vs Control $12.5 \%, p=0.035$ ). One patient with superficial SSI subsequently developed PJI, which required 3 reoperations, and eventually succumbed due to persistent bacteremia. The rate of aseptic wound complications did not differ significantly in both cohorts (ciNPWT 8.3\% Vs Control 2.5\% Vs, $p=0.309$ ).

There were no statistically significant differences in the secondary outcomes which included the length of hospital stay (ciNPWT $31.5 \pm 28.6$ Vs Control $22.9 \pm 19.2$, $p=0.125)$, re-operations due to wound complications (ciNPWT 0\% Vs Control: $2.1 \%, p=1.000$ ), 90-day perioperative mortality (ciNPWT 0\% Vs Control: 2.1\%, $p=1.000)$ and readmission within 30 days of surgery (ciNPWT 0\% Vs Control: 0\%, $p=1.000$ ).

\section{Discussion}

The basic scientific mechanism, and the advantages of ciNPWT were well-reported [22-24]. Immediate effects of ciNPWT include protecting the incision from external contamination, decreasing the lateral tension applied on the incision, increasing the appositional strength, normalizing stress distribution and increasing skin perfusion. Long-term benefits include improving incision quality, in terms of the mechanical strength, histological and gene expression profile.

A recent meta-analysis of 11 studies on ciNPWT in knee or hip arthroplasty (including 8 randomized trials and 3 comparative cohort studies) found that ciNPWT could significantly reduce the incidence of wound complication and SSI in high-risk patients, and patients undergoing revision arthroplasties when compared with conventional dressing [25]. Among the high-risk patients, the ciNPWT cohort had significantly lower rates of wound complication $(\mathrm{OR}=0.38, p=0.030)$ and SSI $(\mathrm{OR}=0.24, p=0.005)$. Likewise, among patients undergoing revision arthroplasty, the ciNPWT cohort had lower rates of wound complication $(\mathrm{OR}=0.33, p<0.001)$ and SSI $(\mathrm{OR}=0.26, p=0.004)$. However, there were no significant differences in wound complication and SSI between ciNPWT versus conventional dressing among non-high-risk patients and patients undergoing primary arthroplasty. Another meta-analysis involving 8 randomized trials reported a significantly lower overall SSI risk for primary and revision arthroplasty in patients having ciNPWT compared to conventional dressings, and specifically lower in revision THA and TKA [26]. However, ciNPWT may increase the risk of noninfectious complications after primary TKA, such as blisters, seroma, hematoma, persistent drainage and wound dehiscence [26].
Existing studies demonstrated the effectiveness of ciNPWT in decreasing wound complications, specifically in revision arthroplasty. In a 2016 comparative study involving high-risk patients with multiple risk factors for SSIs, the ciNPWT group had significantly fewer wound complications $(6.7 \%$ vs $26.9 \%, p=0.024)$ and SSIs $(3.3 \%$ vs $18.5 \%, p=0.045)$ compared to patients treated with antimicrobial dressings [27]. In a 2019 randomised controlled cohort study involving revision arthroplasty in patients with risk factors for wound complications [28], the ciNPWT group had a significantly lower wound complication rate $(10.1 \%$ vs $23.8 \%, p=0.022)$ and rate of re-operation $(2.5 \%$ vs $12.5 \%, p=0.017)$ than the control group (who received conventional dressing). However, ciNPWT was not found to have any significant effect on reducing the number of superficial or deep surgical site infections.

The majority of the literatures studying the use of ciNPWT in arthroplasty were from North America. This might be explained by the current marketing strategies by the industrial companies in targeting the users of ciNPWT devices in the North America. At present, the ciNPWT market was primarily located in North America, with a revenue of USD 738.1 million in 2018 [29]. Nevertheless, the demand for ciNPWT was projected to increase in the Asia-Pacific region, especially In China. The ciNPWT market was expected to grow at a tremendous high rate between 2020 and 2027 in China as the growing economy expediate the market demand for a better dressing material for the postoperative surgical wound management [30].

There is a paucity of clinical data from local and Asian regions. In a recent comparative study in China involving patients undergoing total ankle replacement, the ciNPWT group had lower rates of wound complication 7.7\% vs $19.0 \%, p=0.34)$ and infection $(0 \%$ vs $4.8 \%, p=0.62$, [31], although these differences were not statistically significant. Hence, our study aimed to further investigate the role of ciNPWT in Asian patients undergoing arthroplasty. Our study showed that ciNPWT could reduce the rate of superficial surgical site infections $(0 \%$ vs $12.5 \%$, $p=0.035)$ when compared to conventional dressing. Because of the convincing data showed in our study, ciNPWT is currently used for all patients who undergo revision arthroplasty in our institution.

In our study, it was interesting to note that the overall wound complication rate of the ciNPWT cohort and control cohort did not differ significantly ( $8.3 \%$ vs $14.6 \%$, $p=0.504)$. It was caused by the higher number of noninfectious wound complications in ciNPWT cohort, in particular blister formation. The increase in the blister complication in the surgical wound among ciNPWT cohort might result from inadequate experience in the 
application of ciNPWT during the initial use. Although the difference was not statistically significant, the precautions in the application of ciNPWT to avoid blister formation should be taken. An earlier study using ciNPWT in total knee arthroplasty was prematurely terminated due to blister formation and maceration of the surrounding skin [32]. Vaez-zadeh proposed blisters could form at the interface of the foam edge and the transparent film under the effect of negative pressure, if the skin is not well protected by transparent film in accordance with the manufacturer's recommendations [33]. In our study, a patient was complicated by the blister formation in the ciNPWT cohort on postoperative day 4 (Fig. 3). ciNPWT was stopped, and the conventional dressing was applied. Blisters resolved eventfully without any further complications. To avoid blister formation, the following procedures were recommended during the application of ciNPWT. First, the skin around the surgical wound should be cleaned up and dried thoroughly to ensure no foreign products interfering the negative suction. Second, the foam dressing should be accurately positioned to avoid the foam edge placing directly over edges of the surgical wound because the negative suction force may cause secondary injury to the wound edges. Third, it is important to ensure that the transparent film applied over the foam does not create excessive air spaces or bubbles around the foam dressing, which may affect the pressure distribution on the intact skin around the foam dressing. When the transparent film was applied to the knee, the knee should be flexed around 60 degrees so as to minimize the maceration of the skin upon knee flexion. Fourth, daily checking and documentation of the peri-wound skin condition should be undertaken by the medical and nursing team.

One of the concerns of using ciNPWT was the cost of the device, but it was showed to be cost-effective paradoxically in further studies. The main reasons for hospital readmission after hospital discharge among patients having arthroplasty were infection and wound complication, which contributed $35.9 \%$ and $14.4 \%$ of the unplanned readmissions respectively [34]. By reducing wound complications, ciNPWT could potentially result in cost savings by reducing hospital readmission, and the subsequent surgical management such as reoperation and debridement. In a UK cost-effectiveness analysis on primary hip and knee arthroplasty, an estimated USD1607 per patient could be saved by using a single-use ciNPWT device instead of conventional dressings, with greater savings demonstrated in high-risk subgroups such as patients with obesity and ASA grade $\geq 3$ [35].

There are limitations to this study. First, two models of ciNPWT device, including PICO and Prevena, were applied in the current study, and this may cause

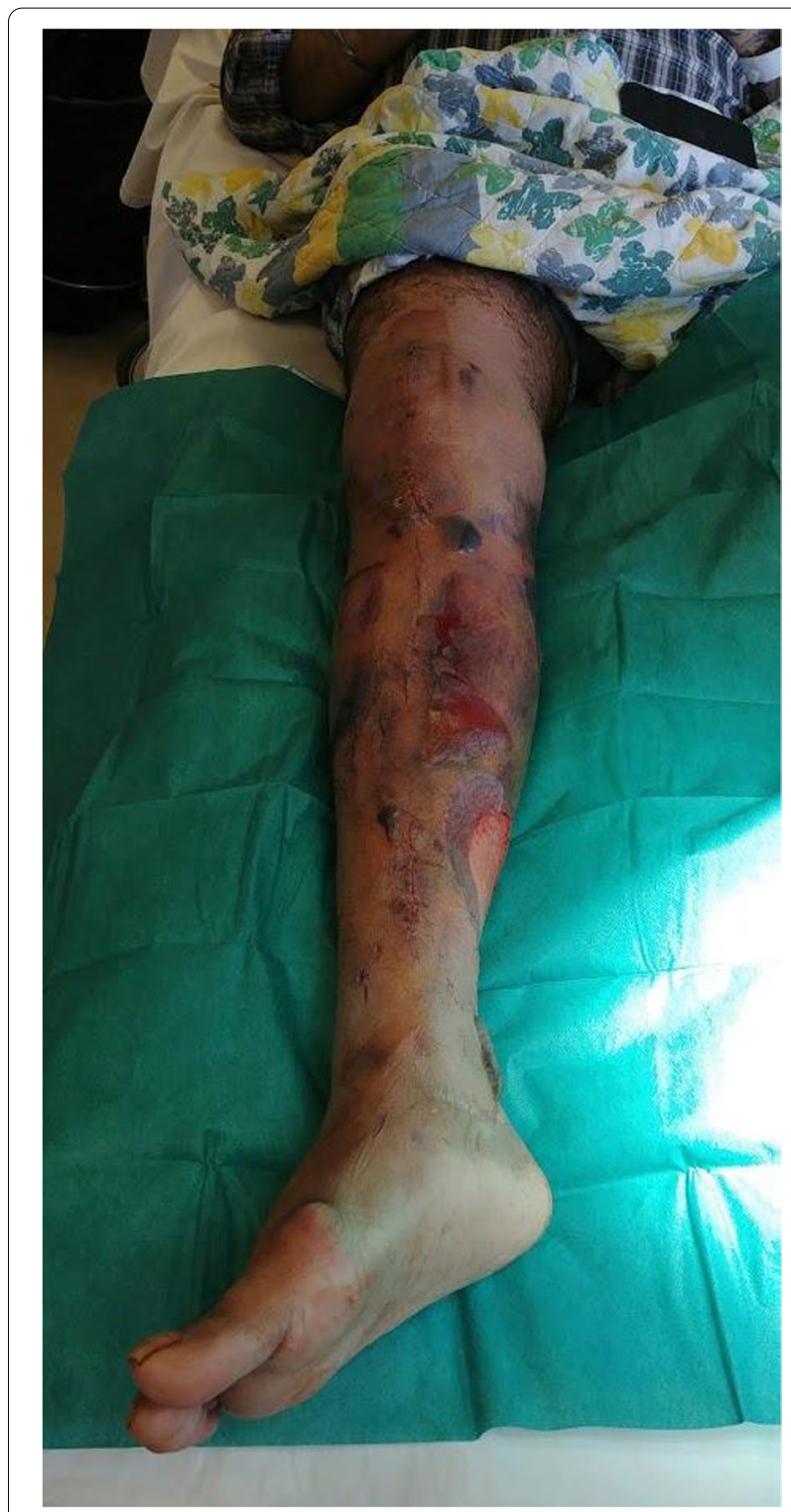

Fig. 3 Blisters were observed medial to surgical incision, which subsequently resolved with conventional dressing

heterogeneity in our results. The prinicipal difference between two devices is the design in foam layers: Prevena having a single layer of foam dressing, while PICO having 4 layers of dressing. Since there was no study demonstrating any significant clinical difference between the two products, they were not analyzed separately in our study. Second, although baseline characteristics of the two cohorts were compared, it is possible that some confounders may be missed due to the retrospective nature of our study. Third, the antibiotic used postoperatively among the septic revision cases were not compared because of the heterogenicity of the 
antibiotic used. This might contribute to the confounding factor. Finally, hip and knee revision arthroplasties, and septic and aseptic revision was not analysed separately in both cohorts because of the insufficient number of revision cases. Additionally, sub-group analysis could be performed in future study involving higher caseloads. Nevertheless, our study was among the first study to investigate the clinical outcome of ciNPWT in Asian patients after revision arthroplasties. Together with existing literature, this work opens to the way towards the use of ciNPWT for wound management in patients undergoing revision arthroplasty or at high risk of surgical site infection.

\section{Conclusion}

The application of ciNPWT could result in a lower rate of superficial surgical site infection when compared with conventional dressing among the patients undergoing revision total knee and total hip arthroplasties.

These results supported the use of ciNPWT in patients undergoing revision arthroplasty. Proper training and precautions should be made during the application of ciNPWT to avoid blister formation.

\begin{abstract}
Abbreviations
ASA: American Society of Anaesthesiologists; CDC: Centers for Disease Control; ciNPWT: Close Incisional Negative Pressure Wound Therapy; ESBL E-coli: Extended Spectrum Beta-Lactamase Escherichia coli; HKU/HA HKW IRB: Institutional Review Board of University of Hong Kong/Hospital Authority Hong Kong West Cluster; MRSA: Methicillin-resistant Staphylococcus aureus; MSSA: Methicillin sensitive Staphylococcus aureus; MSIS: Musculoskeletal Infection Society; NICE: National Institute for Health and Care Excellence; OR: Odds Ratio; PJI: Prosthetic joint infection; RR: Risk Ratio; SSI: Superficial Surgical Site Infection; THA: Total Hip Arthroplasty; TKA: Total Knee Arthroplasty; VRSA: Vancomycin-resistant Staphylococcus aureus.
\end{abstract}

\section{Acknowledgements}

We also acknowledge and express our gratitude to Ms LS Lee for English editing advices.

\section{Authors' contributions}

(1) Concept or design: Ping Keung Chan, (2) Acquisition of data: Wing Chiu Fung, Kar Hei Lam, Winnie Chan, Vincent Wai Kwan Chan. (3) Analysis or interpretation of data: Ping Keung Chan, Wing Chiu Fung, Kar Hei Lam, Henry Fu, Amy Cheung, MH Cheung. (4) Drafting of the article: Ping Keung Chan, Wing Chiu Fung. (5) Critical revision for important intellectual content: Chun Hoi Yan, Kwong Yuen Chiu. All authors had full access to the data, contributed to the study, approved the final version for publication, and take responsibility for its accuracy and integrity.

\section{Funding}

This research received no specific grant from any funding agency in the public, commercial, or not-for-profit sectors.

\section{Availability of data and materials}

The datasets used and/or analysed during the current study are available from the corresponding author on reasonable request.

\section{Declarations}

Ethics approval and consent to participate

Approval from Institutional Review Board of the University of Hong Kong/ Hospital Authority Hong Kong West Cluster (HKU/HA HKW IRB) was obtained for this study (Reference no. UW 21-034). Patient consents were waived by the Institutional Review Board.

\section{Consent for publication}

Not applicable.

\section{Competing interests}

Chun Hoi Yan and Kwong Yuen Chiu are the members of the Editorial Board of Arthroplasty and other authors declare that they have no competing interests. All authors were not involved in the journal's review of or decisions related to this manuscript.

\section{Author details}

'Department of Orthopaedics and Traumatology, Queen Mary Hospital, Hong Kong SAR, China. ${ }^{2}$ Department of Orthopaedics and Traumatology, The University of Hong Kong, Hong Kong SAR, China. ${ }^{3}$ Department of Nursing, Queen Mary Hospital, Operation Theatre Services, Hong Kong SAR, China.

Received: 17 March 2021 Accepted: 16 August 2021

Published online: 03 November 2021

\section{References}

1. Galat DD, McGovern SC, Larson DR, Harrington JR, Hanssen AD, Clarke HD. Surgical treatment of early wound complications following primary total knee arthroplasty. JBJS. 2009;91(1):48-54.

2. Galat DD, McGovern SC, Hanssen AD, Larson DR, Harrington JR, Clarke $\mathrm{HD}$. Early return to surgery for evacuation of a postoperative hematoma after primary total knee arthroplasty. JBJS. 2008:90(11):2331-6.

3. Wagenaar F-CBM, Löwik CAM, Zahar A, Jutte PC, Gehrke T, Parvizi J. Persistent wound drainage after total joint arthroplasty: a narrative review. J Arthroplasty. 2019;34(1):175-82.

4. Grosso MJ, Berg A, LaRussa S, Murtaugh T, Trofa DP, Geller JA. Silverimpregnated occlusive dressing reduces rates of acute periprosthetic joint infection after total joint arthroplasty. J Arthroplasty. 2017;32(3):929-32.

5. DeCarbo WT, Hyer CF. Negative-pressure wound therapy applied to highrisk surgical incisions. J Foot Ankle Surg. 2010;49(3):299-300.

6. Higuera-Rueda CA, Emara AK, Nieves-Malloure Y, Klika AK, Cooper HJ, Cross MB, et al. The Effectiveness of Closed-Incision Negative-Pressure Therapy Versus Silver-Impregnated Dressings in Mitigating Surgical Site Complications in High-Risk Patients After Revision Knee Arthroplasty: The PROMISES Randomized Controlled Trial. J Arthroplasty. 2021;36((7, Supplement)):S295-302 e14.

7. Orgill DP, Bayer LR. Negative pressure wound therapy: past, present and future. Int Wound J. 2013;10(s1):15-9.

8. Morykwas MJ, Argenta LC, Shelton-Brown El, McGuirt W. Vacuum-assisted closure: a new method for wound control and treatment: animal studies and basic foundation. Ann Plast Surg. 1997;38(6):553-62.

9. Huang $C$, Leavitt T, Bayer LR, Orgill DP. Effect of negative pressure wound therapy on wound healing. Curr Probl Surg. 2014;51(7):301-31.

10. Kairinos N, Solomons M, Hudson DA. Negative-pressure wound therapy I: the paradox of negative-pressure wound therapy. Wound Healing Southern Africa. 2017;10(2):6-14.

11. Nam D, Sershon RA, Levine BR, Della Valle CJ. The use of closed incision negative-pressure wound therapy in orthopaedic surgery. J Am Acad Orthop Surg. 2018;26(9):295-302.

12. Mouës CM, van den Bemd GJCM, Heule F, Hovius SER. Comparing conventional gauze therapy to vacuum-assisted closure wound therapy: A prospective randomised trial. Journal of Plastic, Reconstructive \& Aesthetic Surgery. J Plast Reconstr Aesthet Surg. 2007;60(6):672-81.

13. Stannard JP, Robinson JT, Anderson ER, McGwin G Jr, Volgas DA, Alonso JE. Negative Pressure Wound Therapy to Treat Hematomas and Surgical 
Incisions Following High-Energy Trauma. J Trauma Acute Care Surg. 2006;60(6):1301-6.

14. Stannard JP, Singanamala N, Volgas DA. Fix and flap in the era of vacuum suction devices: What do we know in terms of evidence based medicine? Injury. 2010;41(8):780-6.

15. Streubel PN, Stinner DJ, Obremskey WT. Use of Negative-pressure Wound Therapy in Orthopaedic Trauma. J Am Acad Orthop Surg. 2012;20(9):564-74.

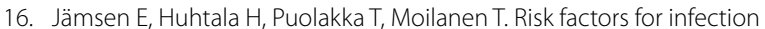
after knee arthroplasty: a register-based analysis of 43,149 Cases. JBJS. 2009;91(1):38-47.

17. Lenguerrand E, Whitehouse MR, Beswick AD, Jones SA, Porter ML, Blom AW. Revision for prosthetic joint infection following hip arthroplasty. Bone Jt Res. 2017:6(6):391-8.

18. Yaghmour KM, Hossain FS, Konan S. Clinical and health-care cost analysis of negative pressure dressing in primary and revision total knee arthroplasty: a systematic review and meta-analysis. JBJS. 2021;103(6):541-8.

19. Pagani NR, Moverman MA, Puzzitiello RN, Menendez ME, Kavolus JJ. The cost-effectiveness ofclosed incisional negative pressure wound therapy for infection prevention after revision total knee arthroplasty. J Knee Surg. 2021.

20. Horan TC, Gaynes RP, Martone WJ, Jarvis WR, Emori TG. CDC definitions of nosocomial surgical site infections, 1992: a modification of CDC definitions of surgical wound infections. Infect Control Hosp Epidemiol. 1992;13(10):606-8.

21. Parvizi J, Zmistowski B, Berbari EF, Bauer TW, Springer BD, Della Valle CJ, et al. New definition for periprosthetic joint infection: from the workgroup of the musculoskeletal infection society. Clin Orthop Relat Res. 2011:469(11):2992.

22. Kilpadi DV, Cunningham MR. Evaluation of closed incision management with negative pressure wound therapy (CIM): hematoma/seroma and involvement of the lymphatic system. Wound Repair Regen. 2011;19(5):588-96.

23. Kilpadi DV, Lessing C, Derrick K. Healed porcine incisions previously treated with a surgical incision management system: mechanical, histomorphometric, and gene expression properties. Aesthetic Plast Surg. 2014;38(4):767-78.

24. Timmers MS, Le Cessie S, Banwell P, Jukema GN. The effects of varying degrees of pressure delivered by negative-pressure wound therapy on skin perfusion. Ann Plast Surg. 2005;55(6):665-71.

25. Kim JH, Lee DH. Are high-risk patient and revision arthroplasty effective indications for closed-incisional negative-pressure wound therapy after total hip or knee arthroplasty? A systematic review and meta-analysis. Int Wound J. 2020;17(5):1310-22

26. Ailaney N, Johns WL, Golladay GJ, Strong B, Kalore NV. Closed Incision Negative Pressure Wound Therapy for Elective Hip and Knee Arthroplasty: A Systematic Review and Meta-Analysis of Randomized Controlled Trials. J Arthroplasty. 2020.

27. Cooper HJ, Bas MA. Closed-incision negative-pressure therapy versus antimicrobial dressings after revision hip and knee surgery: a comparative study. J Arthroplasty. 2016;31(5):1047-52.

28. Newman JM, Siqueira MBP, Klika AK, Molloy RM, Barsoum WK, Higuera CA. Use of closed incisional negative pressure wound therapy after revision total hip and knee arthroplasty in patients at high risk for infection: a prospective. Randomized Clinical Trial J Arthroplasty. 2019;34(3):554-9.e 1.

29. Business F. https://www.fortunebusinessinsights.com/industry-reports/ negative-pressure-wound-therapy-npwt-market-101243. 2018.

30. Bridge D. Asia-Pacific Negative Pressure Wound Therapy Devices Market - Industry Trends and Forecast to 2027. 2020. https://www.databridge marketresearch.com/reports/asia-pacific-negative-pressure-wound-thera py-devices-market.

31. Liu X, Zhang H, Li Y, Chen Y, Deng W, Zhang W. Application of negative pressure wound therapy in total ankle replacement. J Foot Ankle Surg. 2021;60(2):283-7.

32. Howell RD, Hadley S, Strauss E, Pelham FR. Blister formation with negative pressure dressings after total knee arthroplasty. Curr Orthop Pract. 2011;22(2):176-9.

33. Vaez-zadeh $\mathrm{S}$. In response to blister formation with negative pressure dressings. Curr Orthop Pract. 2011;22(6):591.

34. Zmistowski B, Restrepo C, Hess J, Adibi D, Cangoz S, Parvizi J. Unplanned readmission after total joint arthroplasty: rates, reasons, and risk factors. JBJS. 2013;95(20):1869-76.

35. Nherera LM, Trueman P, Karlakki SL. Cost-effectiveness analysis of singleuse negative pressure wound therapy dressings (SNPWT) to reduce surgical site complications (SSC) in routine primary hip and knee replacements. Wound Repair Regen. 2017;25(3):474-82.

\section{Publisher's Note}

Springer Nature remains neutral with regard to jurisdictional claims in published maps and institutional affiliations.
Ready to submit your research? Choose BMC and benefit from:

- fast, convenient online submission

- thorough peer review by experienced researchers in your field

- rapid publication on acceptance

- support for research data, including large and complex data types

- gold Open Access which fosters wider collaboration and increased citations

- maximum visibility for your research: over $100 \mathrm{M}$ website views per year

At BMC, research is always in progress.

Learn more biomedcentral.com/submissions 